\title{
Existence of homoclinic solutions for a class of second-order Hamiltonian systems with subquadratic growth
}

Dan Zhang*

\section{*Correspondence:}

zhang11dan@126.com

Department of Mathematics, Hunan

University of Science and

Engineering, Yongzhou, Hunan

425100, People's Republic of China

\begin{abstract}
By properly constructing a functional and by using the critical point theory, we establish the existence of homoclinic solutions for a class of subquadratic second-order Hamiltonian systems. Our result generalizes and improves some existing ones. An example is given to show that our theorem applies, while the existing results are not applicable.
\end{abstract}

Keywords: homoclinic solutions; critical point theory; Hamiltonian systems; nontrivial solution

\section{Introduction}

Consider the following second-order Hamiltonian system:

$$
\ddot{q}(t)-L(t) q(t)+W_{q}(t, q(t))=0, \quad t \in \mathbb{R},
$$

where $q=\left(q_{1}, q_{2}, \ldots, q_{n}\right) \in \mathbb{R}^{n}, L \in C\left(\mathbb{R}, \mathbb{R}^{n \times n}\right)$ is a symmetric matrix-valued function, and $W(t, q) \in C^{1}\left(\mathbb{R} \times \mathbb{R}^{n}, \mathbb{R}\right), W_{q}(t, q) \in C^{1}\left(\mathbb{R} \times \mathbb{R}^{n}, \mathbb{R}^{n}\right)$ is the gradient of $W$ about $q$. As usual we say that a solution $q(t)$ of (HS) is homoclinic (to 0 ) if $q \in C^{2}\left(\mathbb{R}, \mathbb{R}^{n}\right.$ ) such that $q(t) \rightarrow 0$ and $\dot{q}(t) \rightarrow 0$ as $|t| \rightarrow \infty$. If $q(t) \not \equiv 0, q(t)$ is called a nontrivial homoclinic solution.

By now, the existence and multiplicity of homoclinic solutions for second-order Hamiltonian systems have been extensively investigated in many papers (see, e.g., [1-17] and the references therein) via variational methods. More precisely, many authors studied the existence and multiplicity of homoclinic solutions for (HS); see [5-17]. Some of them treated the case where $L(t)$ and $W(t, u)$ are either independent of $t$ or periodic in $t$ (see, for instance, [5-7]), and a more general case is considered in the recent paper [7]. If $L(t)$ is neither constant nor periodic in $t$, the problem of the existence of homoclinic solutions for (HS) is quite different from the one just described due to the lack of compactness of the Sobolev embedding. After the work of Rabinowitz and Tanaka [8], many results [9-17] were obtained for the case where $L(t)$ is neither constant nor periodic in $t$.

Recently, Zhang and Yuan [15] obtained the existence of a nontrivial homoclinic solution for (HS) by using a standard minimizing argument. In this paper, $(\cdot, \cdot): \mathbb{R}^{n} \times \mathbb{R}^{n} \rightarrow \mathbb{R}$ denotes the standard inner product in $\mathbb{R}^{n}$, and subsequently, $|\cdot|$ is the induced norm. If $q=\left(q_{1}, q_{2}, \ldots, q_{n}\right) \in \mathbb{R}^{n}$, then $|q|=\sqrt{q_{1}^{2}+q_{2}^{2}+\cdots+q_{n}^{2}}$.

๑) 2012 Zhang; licensee Springer. This is an Open Access article distributed under the terms of the Creative Commons Attribution License (http://creativecommons.org/licenses/by/2.0), which permits unrestricted use, distribution, and reproduction in any medium, provided the original work is properly cited. 
Theorem 1.1 (See [15, Theorem 1.1]) Assume that $L$ and $W$ satisfy the following conditions:

(H1) $L(t) \in C\left(\mathbb{R}, \mathbb{R}^{n \times n}\right)$ is a symmetric matrix for all $t \in \mathbb{R}$, and there is a continuous function $\alpha: \mathbb{R} \rightarrow \mathbb{R}$ such that $\alpha(t)>0$ for all $t \in \mathbb{R}$ and $(L(t) q, q) \geq \alpha(t)|q|^{2}$ and $\alpha(t) \rightarrow+\infty$ as $|t| \rightarrow+\infty$.

(H2) $W(t, q)=a(t)|q|^{\gamma}$ where $a(t): \mathbb{R} \rightarrow \mathbb{R}^{+}$is a positive continuous function such that $a(t) \in L^{2}(\mathbb{R}, \mathbb{R}) \cap L^{\frac{2}{2-\gamma}}(\mathbb{R}, \mathbb{R})$ and $1<\gamma<2$ is a constant.

Then (HS) possesses at least one nontrivial homoclinic solution.

In [15-17], the authors considered the case where $W(t, q)$ is subquadratic as $|q| \rightarrow \infty$. However, there are many functions with subquadratic growth but they do not satisfy the condition (H2) in [15] and the corresponding conditions in [16, 17]. For example,

$$
W(t, q)=a(t)|q|^{\gamma}+b(t) \mathrm{e}^{\cos ^{3}|q|}, \quad \forall(t, q) \in\left(\mathbb{R}, \mathbb{R}^{n}\right),
$$

where $1<\gamma<2, a(t), b(t): \mathbb{R} \rightarrow \mathbb{R}^{+}$are positive continuous functions such that $a(t), b(t) \in$ $L^{2}(\mathbb{R}, \mathbb{R}) \cap L^{\frac{2}{2-\gamma}}(\mathbb{R}, \mathbb{R})$.

In this paper, our aim is to revisit (HS) and study the subquadratic case which is not included in [15-17]. Now, we state our main result.

Theorem 1.2 Let the above condition (H1) hold. Moreover, assume that the following conditions hold:

(H3) $W(t, q) \geq a(t)|q|^{\gamma}, \forall(t, q) \in\left(\mathbb{R}, \mathbb{R}^{n}\right)$, where $a(t): \mathbb{R} \rightarrow \mathbb{R}^{+}$is a positive continuous function such that $a(t) \in L^{2}(\mathbb{R}, \mathbb{R}) \cap L^{\frac{2}{2-\gamma}}(\mathbb{R}, \mathbb{R})$ and $1<\gamma<2$ is a constant.

(H4) $\left|W_{q}(t, q)\right| \leq f_{1}(t)|q|^{\gamma-1}+f_{2}(t), \forall(t, q) \in\left(\mathbb{R}, \mathbb{R}^{n}\right)$ where $f_{1}(t), f_{2}(t): \mathbb{R} \rightarrow \mathbb{R}^{+}$are positive continuous functions such that $f_{1}(t), f_{2}(t) \in L^{2}(\mathbb{R}, \mathbb{R}) \cap L^{\frac{2}{2-\gamma}}(\mathbb{R}, \mathbb{R})$.

Then (HS) possesses at least one nontrivial homoclinic solution.

Remark 1.1 Obviously, the condition (H2) is a special case of (H3)-(H4). If (H2) holds, so do (H3)-(H4); however, the reverse is not true. $W(t, q)$ defined in (1) can satisfy the conditions (H3) and (H4), but $W(t, q)$ cannot satisfy the condition (H2). So, we generalize and significantly improve Theorem 1.1 in [15].

Remark 1.2 We still consider the function $W(t, q)$ defined in (1),

$$
W(t, q) \geq a(t)|q|^{\gamma}+b(t) \mathrm{e}^{-1}, \quad \forall(t, q) \in\left(\mathbb{R}, \mathbb{R}^{n}\right) .
$$

Due to $\inf _{t \in \mathbb{R}} a(t)=0$, there are no constants $b, r_{1}>0$ such that

$$
W(t, q) \geq b|q|^{\gamma}, \quad \forall t \in \mathbb{R} \text { and }|q| \geq r_{1},
$$

so $W(t, q)$ does not satisfy the conditions (W2) and (W3) in [16]. Moreover, for any given $1<\gamma<\frac{3}{2}, W(t, q)$ does not satisfy the condition (W2) in [17]. Therefore, we also extend Theorem 1.2 in [16] and Theorem 1.1 in [17].

Example 1.1 Consider the following second-order Hamiltonian system with $n=3$ :

$$
\ddot{q}-L(t) q+W_{q}(t, q)=0, \quad \forall t \in \mathbb{R}
$$


where

$$
L(t)=\left(\begin{array}{ccc}
2+t^{2} & 0 & 0 \\
0 & 2+t^{2} & 0 \\
0 & 0 & 2+t^{2}
\end{array}\right), \quad W(t, q)=\left(\frac{1}{1+|t|^{3}}\right)|q|^{\frac{5}{4}}+\left(\frac{1}{1+|t|^{2}}\right) \mathrm{e}^{\sin ^{3}|q|}
$$

Let $\alpha(t)=t^{2}, \gamma=\frac{5}{4}$ and $a_{1}(t)=\frac{1}{1+|t|^{3}}, a_{2}(t)=\frac{1}{1+|t|^{2}}, W_{q}(t, q)=\frac{5}{4} a_{1}(t)|q|^{-\frac{3}{4}} q+3 a_{2}(t) \mathrm{e}^{\sin ^{3}|q|} \times$ $|q|^{-1} q \sin ^{2}|q| \cos |q| \leq \frac{5}{4} a_{1}(t)|q|^{\frac{1}{4}}+3 a_{2}(t)$ e, $W_{q}(t, 0)=0$. Clearly, (H1), (H3), and (H4) hold. Therefore, by applying Theorem 1.2, the Hamiltonian system (2) possesses at least one nontrivial homoclinic solution.

Remark 1.3 It is easy to see that (H2) in Theorem 1.1 is not satisfied, so we cannot obtain the existence of homoclinic solutions for the Hamiltonian system (2) by Theorem 1.1. On the other hand, $W$ does not satisfy the conditions (W2) and (W5) of [17], then we cannot obtain the existence of homoclinic solutions for the Hamiltonian system (2) by Theorem 1.1 in [17].

The remainder of this paper is organized as follows. In Section 2, some preliminary results are presented. In Section 3, we give the proof of Theorem 1.2.

\section{Preliminary results}

In order to establish our result via the critical point theory, we firstly describe some properties of the space on which the variational associated with (HS) is defined. Like in [15], let

$$
E=\left\{q \in H^{1}\left(\mathbb{R}, \mathbb{R}^{n}\right): \int_{\mathbb{R}}\left[|\dot{q}|^{2}+(L(t) q(t), q(t))\right] d t<\infty\right\} .
$$

Then the space $E$ is a Hilbert space with the inner product

$$
\langle x, y\rangle=\int_{\mathbb{R}}[(\dot{x}(t), \dot{y}(t))+(L(t) x(t), y(t))] d t
$$

and the corresponding norm $\|x\|^{2}=\langle x, x\rangle$. Note that

$$
E \subset H^{1}\left(\mathbb{R}, \mathbb{R}^{n}\right) \subset L^{p}\left(\mathbb{R}, \mathbb{R}^{n}\right)
$$

for all $p \in[2,+\infty)$ with the embedding being continuous. Here $L^{p}\left(\mathbb{R}, \mathbb{R}^{n}\right)(2 \leq p<+\infty)$ and $H^{1}\left(\mathbb{R}, \mathbb{R}^{n}\right)$ denote the Banach spaces of functions on $\mathbb{R}$ with values in $\mathbb{R}^{n}$ under the norms

$$
\|q\|_{p}:=\left(\int_{\mathbb{R}}|q|^{p} d t\right)^{1 / p}
$$

and

$$
\|q\|_{H^{1}}:=\left(\|q\|_{2}^{2}+\|\dot{q}\|_{2}^{2}\right)^{1 / 2}
$$


respectively. In particular, for $p=+\infty$, there exists a constant $C>0$ such that

$$
\|q\|_{\infty} \leq C\|q\|, \quad \forall q \in E,
$$

here $\|q\|_{\infty}:=\operatorname{ess} \sup \{|q(t)|: t \in \mathbb{R}\}$.

Lemma 2.1 There exists a constant $\beta>0$ such that if $q \in E$, then

$$
\|q\| \geq \sqrt{\beta}\|q\|_{2} .
$$

Proof From (H1), we can imply that there exists a constant $\beta>0$ such that

$$
(L(t) q, q) \geq \beta|q|^{2}
$$

for all $t \in \mathbb{R}$ and $q \in \mathbb{R}^{n}$. By the above inequality, one has

$$
\|q\|^{2} \geq \int_{\mathbb{R}}(L(t) q(t), q(t)) d t \geq \beta \int_{\mathbb{R}}|q(t)|^{2} d t=\beta\|q\|_{2}^{2} .
$$

So, the lemma is proved.

Lemma 2.2 ([9, Lemma 1]) Suppose that L satisfies (H1). Then the embedding of $E$ in $L^{2}\left(\mathbb{R}, \mathbb{R}^{n}\right)$ is compact.

Lemma 2.3 Suppose that (H1) and (H4) are satisfied. If $q_{k} \rightarrow q$ (weakly) in E, then $W_{q}\left(t, q_{k}\right) \rightarrow W_{q}(t, q)$ in $L^{2}\left(\mathbb{R}, \mathbb{R}^{n}\right)$.

Proof Assume that $q_{k} \rightarrow q$ in $E$. Then there exists a constant $d_{1}>0$ such that, by the Banach-Steinhaus theorem and (3),

$$
\sup _{k \in N}\left\|q_{k}\right\|_{\infty} \leq d_{1}, \quad\|q\|_{\infty} \leq d_{1} .
$$

Since $1<\gamma<2$, by $(\mathrm{H} 4)$ there exists a constant $d_{2}>0$ such that

$$
\left|W_{q}\left(t, q_{k}\right)\right| \leq d_{2} f_{1}(t)+f_{2}(t), \quad\left|W_{q}(t, q)\right| \leq d_{2} f_{1}(t)+f_{2}(t)
$$

for all $k \in N$ and $t \in \mathbb{R}$. Hence,

$$
\left|W_{q}\left(t, q_{k}\right)-W_{q}(t, q)\right| \leq 2 d_{2} f_{1}(t)+2 f_{2}(t) .
$$

On the other hand, by Lemma 2.2, $q_{k} \rightarrow q$ in $L^{2}$, passing to a subsequence if necessary, which implies $q_{k}(t) \rightarrow q(t)$ for almost every $t \in \mathbb{R}$. Then using Lebesgue's convergence theorem, the lemma is proved.

Now, we introduce more notation and some necessary definitions. Let $E$ be a real Banach space, $I \in C^{1}(E, \mathbb{R})$, which means that $I$ is a continuously Fréchet-differentiable functional defined on $E$. Recall that $I \in C^{1}(E, \mathbb{R})$ is said to satisfy the (PS) condition if any sequence $\left\{u_{j}\right\}_{j \in N} \subset E$, for which $\left\{I\left(u_{j}\right)\right\}_{j \in N}$ is bounded and $I^{\prime}\left(u_{j}\right) \rightarrow 0$ as $j \rightarrow+\infty$, possesses a convergent subsequence in $E$. 
Lemma 2.4 ([18, Theorem 2.7]) Let E be a real Banach space, and let us have $I \in C^{1}(E, \mathbb{R})$ satisfying the (PS) condition. If I is bounded from below, then

$$
c \equiv \inf _{E} I
$$

is a critical value of $I$.

\section{Proof of Theorem 1.2}

Now, we are going to establish the corresponding variational framework to obtain homoclinic solutions of (HS). Define the functional $I: E \rightarrow \mathbb{R}$

$$
\begin{aligned}
I(q) & =\int_{\mathbb{R}}\left[\frac{1}{2}|\dot{q}(t)|^{2}+\frac{1}{2}(L(t) q(t), q(t))-W(t, q(t))\right] d t \\
& =\frac{1}{2}\|q\|^{2}-\int_{\mathbb{R}} W(t, q(t)) d t .
\end{aligned}
$$

Lemma 3.1 Under the assumptions of Theorem 1.2, we have

$$
I^{\prime}(q) v=\int_{\mathbb{R}}\left[(\dot{q}(t), \dot{v}(t))+(L(t) q(t), v(t))-\left(W_{q}(t, q(t)), v(t)\right)\right] d t,
$$

which yields that

$$
I^{\prime}(q) q=\|q\|^{2}-\int_{\mathbb{R}}\left(W_{q}(t, q(t)), q(t)\right) d t .
$$

Moreover, I is a continuously Fréchet-differentiable functional defined on E, i.e., I $\in$ $C^{1}(E, \mathbb{R})$ and any critical point of $I$ on $E$ is a classical solution of $(\mathrm{HS})$ with $q( \pm \infty)=0=$ $\dot{q}( \pm \infty)$.

Proof We firstly show that $I \in C^{1}(E, \mathbb{R})$. Let $q \in E$, by (3), (H4), and the Hölder inequality, we have

$$
\begin{aligned}
0 \leq & \int_{\mathbb{R}} W(t, q(t)) d t \leq \int_{\mathbb{R}}\left(f_{1}(t)|q(t)|^{\gamma}+f_{2}(t)|q(t)|\right) d t \\
\leq & \left(\int_{\mathbb{R}}\left|f_{1}(t)\right|^{\frac{2}{2-\gamma}} d t\right)^{\frac{2-\gamma}{2}}\left(\int_{\mathbb{R}}|q(t)|^{\gamma \cdot \frac{2}{\gamma}} d t\right)^{\frac{\gamma}{2}} \\
& +\left(\int_{\mathbb{R}}\left|f_{2}(t)\right|^{2} d t\right)^{\frac{1}{2}}\left(\int_{\mathbb{R}}|q(t)|^{2} d t\right)^{\frac{1}{2}} \\
= & \left\|f_{1}\right\|_{\frac{2}{2-\gamma}}\|q\|_{2}^{\gamma}+\left\|f_{2}\right\|_{2}\|q\|_{2} \\
\leq & \frac{1}{(\sqrt{\beta})^{\gamma}}\left\|f_{1}\right\|_{\frac{2}{2-\gamma}}\|q\|^{\gamma}+\frac{1}{\sqrt{\beta}}\left\|f_{2}\right\|_{2}\|q\|<+\infty .
\end{aligned}
$$

Combining (5) and (8), we show that $I: E \rightarrow \mathbb{R}$. Next, we prove that $I \in C^{1}(E, \mathbb{R})$. Rewrite $I$ as follows:

$$
I=I_{1}-I_{2}
$$


where

$$
I_{1}:=\int_{\mathbb{R}}\left[\frac{1}{2}|\dot{q}(t)|^{2}+\frac{1}{2}(L(t) q(t), q(t))\right] d t, \quad I_{2}:=\int_{\mathbb{R}} W(t, q(t)) d t .
$$

It is easy to check that $I_{1} \in C^{1}(E, \mathbb{R})$ and

$$
I_{1}^{\prime}(q) v=\int_{\mathbb{R}}[(\dot{q}(t), \dot{v}(t))+(L(t) q(t), v(t))] d t .
$$

Thus, it is sufficient to show that this is the case for $I_{2}$. In the process we will see that

$$
I_{2}^{\prime}(q) v=\int_{\mathbb{R}}\left(W_{q}(t, q(t)), v(t)\right) d t,
$$

which is defined for all $q, v \in E$. For any given $q \in E$, let us define $J(q): E \rightarrow \mathbb{R}$ as follows:

$$
J(q) v=\int_{\mathbb{R}}\left(W_{q}(t, q(t)), v(t)\right) d t, \quad v \in E .
$$

It is obvious that $J(q)$ is linear. Now, we show that $J(q)$ is bounded. Indeed, for any given $q \in E$, by (3) and (H4), there exists a constant $d_{3}>0$ such that

$$
\left|W_{q}(t, q(t))\right| \leq f_{1}(t)|q|^{\gamma-1}+f_{2}(t) \leq d_{3} f_{1}(t)+f_{2}(t)
$$

for all $t \in \mathbb{R}$, which yields that by (4) and the Hölder inequality,

$$
\begin{aligned}
|J(q) v| & =\left|\int_{\mathbb{R}}\left(W_{q}(t, q(t)), v(t)\right) d t\right| \leq \int_{\mathbb{R}}\left[d_{3} f_{1}(t)|v(t)|+f_{2}(t)|v(t)|\right] d t \\
& \leq d_{3}\left\|f_{1}\right\|_{2}\|v\|_{2}+\left\|f_{2}\right\|_{2}\|v\|_{2} \leq \frac{1}{\sqrt{\beta}}\left(d_{3}\left\|f_{1}\right\|_{2}+\left\|f_{2}\right\|_{2}\right)\|v\| .
\end{aligned}
$$

Moreover, for any $q, v \in E$, by the mean value theorem, we have

$$
\int_{\mathbb{R}} W(t, q(t)+v(t)) d t-\int_{\mathbb{R}} W(t, q(t)) d t=\int_{\mathbb{R}}\left(W_{q}(t, q(t)+h(t) v(t)), v(t)\right) d t,
$$

where $h(t) \in(0,1)$. Therefore, by Lemma 2.3 and the Hölder inequality, one has

$$
\begin{gathered}
\int_{\mathbb{R}}\left(W_{q}(t, q(t)+h(t) v(t)), v(t)\right) d t-\int_{\mathbb{R}}\left(W_{q}(t, q(t)), v(t)\right) d t \\
=\int_{\mathbb{R}}\left(W_{q}(t, q(t)+h(t) v(t))-W_{q}(t, q(t)), v(t)\right) d t \rightarrow 0
\end{gathered}
$$

as $v \rightarrow 0$ in $E$. Combining (11) and (12), we see that (10) holds. It remains to prove that $I_{2}^{\prime}$ is continuous. Suppose that $q \rightarrow q_{0}$ in $E$ and note that

$$
I_{2}^{\prime}(q) v-I_{2}^{\prime}\left(q_{0}\right) v=\int_{\mathbb{R}}\left(W_{q}(t, q(t))-W_{q}\left(t, q_{0}(t)\right), v(t)\right) d t .
$$

By Lemma 2.3 and the Hölder inequality, we obtain that

$$
I_{2}^{\prime}(q) v-I_{2}^{\prime}\left(q_{0}\right) v \rightarrow 0
$$

as $q \rightarrow q_{0}$, which implies the continuity of $I_{2}^{\prime}$ and $I \in C^{1}(E, \mathbb{R})$. 
Lastly, we check that critical points of $I$ are classical solutions of (HS) satisfying $q(t) \rightarrow 0$ and $\dot{q}(t) \rightarrow 0$ as $|t| \rightarrow+\infty$. We know that $E \subset H^{1}\left(\mathbb{R}, \mathbb{R}^{n}\right) \subset C^{0}\left(\mathbb{R}, \mathbb{R}^{n}\right)$, the space of continuous functions $q$ on $\mathbb{R}$ such that $q(t) \rightarrow 0$ as $|t| \rightarrow+\infty$. Moreover, if $q$ is one critical point of $I$, by (6) we have

$$
\ddot{q}(t)=L(t) q-W_{q}(t, q)
$$

which yields that $q \in C^{2}\left(\mathbb{R}, \mathbb{R}^{n}\right)$, i.e., $q$ is a classical solution of (HS). Since $q$ is one critical point of $I$, we have

$$
I^{\prime}(q) q=\int_{\mathbb{R}}\left[(\dot{q}(t), \dot{q}(t))+(L(t) q(t), q(t))-\left(W_{q}(t, q(t)), q(t)\right)\right] d t=0 .
$$

It follows from $q(t) \rightarrow 0$ as $|t| \rightarrow+\infty$ and the above equality that

$$
\int_{\mathbb{R}}(\dot{q}(t), \dot{q}(t)) d t \rightarrow 0, \quad \text { as }|t| \rightarrow+\infty
$$

Hence, $q$ satisfies $\dot{q}(t) \rightarrow 0$ as $|t| \rightarrow+\infty$. This proof is complete.

Lemma 3.2 Under the assumptions of Theorem 1.2, I satisfies the (PS) condition.

Proof In fact, assume that $\left\{q_{j}\right\}_{j \in N} \subset E$ is a sequence such that $\left\{I\left(q_{j}\right)\right\}_{j \in N}$ is bounded and $I^{\prime}\left(q_{j}\right) \rightarrow 0$ as $j \rightarrow \infty$. Then there exists a constant $C_{1}>0$ such that

$$
\left|I\left(q_{j}\right)\right| \leq C_{1}, \quad\left\|I^{\prime}\left(q_{j}\right)\right\|_{E^{*}} \leq C_{1}
$$

for every $j \in N$.

We firstly prove that $\left\{q_{j}\right\}_{j \in N}$ is bounded in $E$. By (5) and (8), we have

$$
\begin{aligned}
\frac{1}{2}\left\|q_{j}\right\|^{2} & =I\left(q_{j}\right)+\int_{\mathbb{R}} W\left(t, q_{j}(t)\right) d t \\
& \leq C_{1}+\frac{1}{(\sqrt{\beta})^{\gamma}}\left\|f_{1}\right\|_{\frac{2}{2-\gamma}}\left\|q_{j}\right\|^{\gamma}+\frac{1}{\sqrt{\beta}}\left\|f_{2}\right\|_{2}\left\|q_{j}\right\| .
\end{aligned}
$$

Combining (13) and (14), we obtain that

$$
\frac{1}{2}\left\|q_{j}\right\|^{2}-\frac{1}{(\sqrt{\beta})^{\gamma}}\left\|f_{1}\right\|_{\frac{2}{2-\gamma}}\left\|q_{j}\right\|^{\gamma}-\frac{1}{\sqrt{\beta}}\left\|f_{2}\right\|_{2}\left\|q_{j}\right\| \leq C_{1} .
$$

Since $1<\gamma<2$, the above inequality shows that $\left\{q_{j}\right\}_{j \in N}$ is bounded in $E$. By Lemma 2.2, the sequence $\left\{q_{j}\right\}_{j \in N}$ has a subsequence, again denoted by $\left\{q_{j}\right\}_{j \in N}$, and there exists $q \in E$ such that

$$
\begin{aligned}
& q_{j} \rightarrow q, \text { weakly in } E, \\
& q_{j} \rightarrow q, \text { strongly in } L^{2}\left(\mathbb{R}, \mathbb{R}^{n}\right) .
\end{aligned}
$$

Hence,

$$
\left(I^{\prime}\left(q_{j}\right)-I^{\prime}(q), q_{j}-q\right) \rightarrow 0
$$




$$
\int_{\mathbb{R}}\left(W_{q}\left(t, q_{j}(t)\right)-W_{q}(t, q(t)), q_{j}(t)-q(t)\right) d t \rightarrow 0
$$

as $j \rightarrow+\infty$. Moreover, an easy computation shows that

$$
\begin{aligned}
& \left(I^{\prime}\left(q_{j}\right)-I^{\prime}(q), q_{j}-q\right) \\
& \quad=\left\|q_{j}-q\right\|^{2}-\int_{\mathbb{R}}\left(W_{q}\left(t, q_{j}(t)\right)-W_{q}(t, q(t)), q_{j}(t)-q(t)\right) d t
\end{aligned}
$$

So, $\left\|q_{j}-q\right\| \rightarrow 0$ as $j \rightarrow+\infty$, i.e., $I$ satisfies the Palais-Smale condition.

Now, we can give the proof of Theorem 1.2.

Proof of Theorem 1.2 By (5) and (8), for every $r \in \mathbb{R} \backslash\{0\}$ and $q \in E \backslash\{0\}$, we have

$$
\begin{aligned}
I(r q) & =\frac{r^{2}}{2}\|q\|^{2}-\int_{\mathbb{R}} W(t, r q(t)) d t \\
& \geq \frac{r^{2}}{2}\|q\|^{2}-\int_{\mathbb{R}}\left[f_{1}(t)|r q(t)|^{\gamma}+f_{2}(t)|r q(t)|\right] d t \\
& \geq \frac{r^{2}}{2}\|q\|^{2}-|r|^{\gamma} \frac{1}{(\sqrt{\beta})^{\gamma}}\left\|f_{1}\right\|_{\frac{2}{2-\gamma}}\|q\|^{\gamma}-|r| \frac{1}{\sqrt{\beta}}\left\|f_{2}\right\|_{2}\|q\| .
\end{aligned}
$$

Since $1<\gamma<2$, (16) implies that $I(r q) \rightarrow+\infty$ as $|r| \rightarrow+\infty$. Consequently, $I$ is a functional bounded from below. By Lemmas 3.2 and 2.4, I possesses a critical value $c=\inf _{q \in E} I(q)$, i.e., there is a $q \in E$ such that

$$
I(q)=c, \quad I^{\prime}(q)=0
$$

On the other hand, take $c_{0} \in \mathbb{R}^{n}$ with $\left|c_{0}\right| \neq 0$, and let $\varphi \in E$ be given by

$$
\varphi(t)= \begin{cases}c_{0} \sin \left(\frac{\pi}{t_{2}-t_{1}}\left(t-t_{1}\right)\right) & \text { if } t \in\left[t_{1}, t_{2}\right], \\ 0 & \text { if } t \in \mathbb{R} \backslash\left[t_{1}, t_{2}\right]\end{cases}
$$

where $-\infty<t_{1}<t_{2}<+\infty$. Then we obtain that

$$
\begin{aligned}
I(r \varphi) & =\frac{r^{2}}{2}\|\varphi\|^{2}-\int_{\mathbb{R}} W(t, r \varphi(t)) d t \\
& \leq \frac{r^{2}}{2}\|\varphi\|^{2}-|r|^{\gamma} \int_{\mathbb{R}} a(t)|\varphi(t)|^{\gamma} d t,
\end{aligned}
$$

which yields that $I(r \varphi)<0$ as $|r|$ small enough since $1<\gamma<2$, i.e., the critical point obtained above is nontrivial. 


\section{Acknowledgements}

This work is supported by the Research Foundation of Education Bureau of Hunan Province, China (No.11C0594). The authors would like to thank the anonymous referees very much for helpful comments and suggestions which led to the improvement of presentation and quality of the work.

Received: 6 July 2012 Accepted: 25 October 2012 Published: 13 November 2012

\section{References}

1. Tang, C: Periodic solutions for nonautonomous second order systems with sublinear nonlinearity. Proc. Am. Math. Soc. 126, 3263-3270 (1998)

2. Ambrosetti, A, Coti Zelati, V: Multiple homoclinic orbits for a class of conservative systems. Rend. Semin. Mat. Univ. Padova 89, 177-194 (1993)

3. Zhang, X, Zhou, Y: Periodic solutions of non-autonomous second order Hamiltonian systems. J. Math. Anal. Appl. 345 929-933 (2008)

4. Cordaro, G, Rao, G: Three periodic solutions for perturbed second order Hamiltonian systems. J. Math. Anal. Appl. 359 780-785 (2009)

5. Bonanno, G, Livrea, R: Multiple periodic solutions for Hamiltonian systems with not coercive potential. J. Math. Anal. Appl. 363, 627-638 (2010)

6. Paturel, E: Multiple homoclinic orbits for a class of Hamiltonian systems. Calc. Var. Partial Differ. Equ. 12, 117-143 (2001)

7. Izydorek, M, Janczewska, J: Homoclinic solutions for a class of second order Hamiltonian systems. J. Differ. Equ. 219, 375-389 (2005)

8. Rabinowitz, PH, Tanaka, K: Some results on connecting orbits for a class of Hamiltonian systems. Math. Z. 206 473-499 (1991)

9. Omana, W, Willem, M: Homoclinic orbits for a class of Hamiltonian systems. Differ. Integral Equ. 5, 1115-1120 (1992)

10. Ou, Z, Tang, C: Existence of homoclinic solutions for the second order Hamiltonian systems. J. Math. Anal. Appl. 291, 203-213 (2004)

11. Tang, X, Xiao, L: Homoclinic solutions for non-autonomous second-order Hamiltonian systems with a coercive potential. J. Math. Anal. Appl. 351, 586-594 (2009)

12. Tang, $X$, Lin, $X$ : Homoclinic solutions for a class of second order Hamiltonian systems. J. Math. Anal. Appl. 354, 539-549 (2009)

13. Yang, J, Zhang, F: Infinitely many homoclinic orbits for the second-order Hamiltonian systems with super-quadratic potentials. Nonlinear Anal., Real World Appl. 10, 1417-1423 (2009)

14. Zhang, Z, Yuan, R: Homoclinic solutions for some second-order non-autonomous systems. Nonlinear Anal. 71, 5790-5798 (2009)

15. Zhang, Z, Yuan, R: Homoclinic solutions for a class of non-autonomous subquadratic second-order Hamiltonian systems. Nonlinear Anal. 71, 4125-4130 (2009)

16. Ding, Y: Existence and multiplicity results for homoclinic solutions to a class of Hamiltonian systems. Nonlinear Anal. 25, 1095-1113 (1995)

17. Zhang, Q, Liu, C: Infinitely many homoclinic solutions for second order Hamiltonian systems. Nonlinear Anal. 72 894-903 (2010)

18. Rabinowitz, PH: Minimax Methods in Critical Point Theory with Applications to Differential Equations. CBMS Regional Conference Series in Mathematics, vol. 65. Am. Math. Soc., Providence (1986)

\section{Submit your manuscript to a SpringerOpen ${ }^{\ominus}$ journal and benefit from:}

- Convenient online submission

- Rigorous peer review

- Immediate publication on acceptance

- Open access: articles freely available online

- High visibility within the field

- Retaining the copyright to your article 